\title{
Weakly Nonlinear Wavepackets in the Korteweg-deVries Equation: The KdV/NLS Connection
}

\author{
John P. Boyd \\ Dept. of Atmospheric, Oceanic and Space Science and Program in Scientific \\ Computing, 2455 Hayward Avenue, Ann Arbor MI 48109, \\ (jpboyd@engin.umich.edu; http://www-personal.engin.umich.edu:/ \\ jpboyd/; Phone: (734) 764-3338; FAX: (734) 764-5137). \\ Guan-yu Chen \\ Institute of Harbour and Marine Technology \\ Wuchi, Taichung 435 \\ Taiwan \\ email: gychen@mail.ihmt.gov.tw
}

Keywords: envelope solitary wave, KdV equation

\begin{abstract}
If the initial condition for the Korteweg-deVries (KdV) equation is a weakly nonlinear wavepacket, then its evolution is described by the Nonlinear Schrödinger (NLS) equation. This KdV/NLS connection has been known for many years, but its various aspects and implications have been discussed only in asides. In this note, we attempt a more focused and comprehensive discussion including such as issues as the $\mathrm{KdV}$-induced long wave pole in the nonlinear coefficient of the NLS equation, the derivation of NLS from KdV through perturbation theory, resonant effects that give the NLS equation a wide range of applicability, and numerical illustrations. The multiple scales/nonlinear perturbation theory is explicitly extended to two orders beyond that which yields the NLS equation; the wave envelope evolves under a Generalized-NLS equation which is third order in space and quintically-nonlinear.
\end{abstract}

1 This work was supported by the National Science Foundation through grant OCE9521133. Dr. Chen is grateful for a three-year graduate fellowship provided by the Department of Education, Taiwan, R. O. C. We also thank Prof. Thiab Taha and the other organizers of the IMACS 1999 conference on "Nonlinear Evolution Equations and Wave Phenomena: Computation and Theory". 


\section{Introduction}

Johnson $[5,6]$ showed that in the long wave limit, the dynamics of wavepackets in the Korteweg-deVries (KdV) equation could be described by the Nonlinear Schrödinger (NLS) equation. Marshall and Boyd[8] wrote a program to compute the NLS coefficients for equatorially-trapped planetary-scale ocean waves and then derived the KdV coefficients directly from those of the NLS equation. One could doubtless find a plethora of similar examples by a thorough literature search.

However, in these previous articles, the KdV/NLS relationship has been a mere parenthesis of much more comprehensive and far-ranging studies. (The exception has been the work of Tracy, Larson, Osborne and Bergamasco[10,11], who give a very readable analysis of the connection between the NLS and $\mathrm{KdV}$ periodic inverse scattering methods for wavepackets; their methods and conclusions are complementary to ours.) In this note, using a mixture of review and original computations, we wish to make the NLS/KdV connection the primary theme.

In particular, we shall extend the weakly nonlinear/multiple scales theory to two orders beyond the NLS equation. We shall further test the theory through high-resolution numerical experiments.

\section{Linear Wavepackets}

To understand the form of the multiple scales expansion for nonlinear flow, it is helpful to first understand the simpler case of infinitesimal amplitude ("linear") waves. In one space dimension in a spatially-uniform medium without boundaries, a wave disturbance can always be represented as a sum of Fourier integrals, one for each branch of the dispersion relation, of the form[12]

$$
u(x, t)=\frac{1}{2 \pi} \int_{-\infty}^{\infty} U(\tilde{k}) \exp (i \tilde{k} x-i w(\tilde{k}) t) d \tilde{k}
$$

where $w(\tilde{k})$ is the dispersion relation for a given branch. For a wavepacket in the narrow sense, as used here, the Fourier transform amplitude is sharply peaked about some wavenumber $\tilde{k}=k$. Without approximation, the integral can be rewritten as the product of a "carrier wave" factor,

$$
E(x, t) \equiv \exp (i[k x-\omega(k) t])
$$


and an "envelope" $A\left(x-c_{g} t, t\right)$ where

$$
c_{g}(k) \equiv \frac{\partial w}{\partial k}(k), \quad[\text { Group velocity }]
$$

Introducing a spatial coordinate in a frame of reference moving at the group velocity,

$$
\zeta \equiv x-c_{g} t
$$

the wavepacket is

$$
u(x, t)=E(x, t) A(\zeta, t)
$$

where $A(\zeta, t)$ is given exactly by

$$
A(\zeta, t)=\frac{1}{2 \pi} \int_{-\infty}^{\infty} \alpha(\kappa) \exp (i \kappa \zeta-i \Omega(\kappa) t) d \kappa
$$

The Fourier amplitude is

$$
\alpha(\kappa) \equiv U(k+\kappa)
$$

and the new frequency is

$$
\Omega(\kappa) \equiv w(k+\kappa)-w(k)-c_{g}(k) \kappa
$$

The derivation is merely a matter of splitting off the first two terms in the Taylor series of $w(k+\kappa)$ about $k$.

The crucial point is that the envelope is described by a Fourier integral of the exactly the same form as $u(x, t)$ itself except that the spatial coordinate is now in a frame of reference which is travelling at the group velocity and the amplitude and frequency functions have been redefined.

Because $\alpha(\kappa)$ is sharply peaked about $\kappa=0$ if the envelope is sufficiently wide in $\zeta$, it is legitimate to expand the frequency in a power series in $\kappa$ and retain only the first few terms. This is equivalent to the multiple scales expansion of the next section where the perturbation is the ratio of the narrow scale of the carrier wave, $1 / k$, with the broad scale of the envelope. The approximation of Taylor-expanding $\Omega$ up to a finite order is equivalent, without additional approximations, to replacing the true wave equation satisfied by $A(\zeta, t)$ with the approximation

$$
A_{t}-i \frac{1}{2} w_{k k}(k) A_{\zeta \zeta}-\frac{1}{6} w_{k k k}(k) A_{\zeta \zeta \zeta}+i \frac{1}{24} w_{k k k k}(k) A_{\zeta \zeta \zeta \zeta}+\frac{1}{120} w_{k k k k k}(k) A_{\zeta \zeta \zeta \zeta \zeta}=0
$$


Example: Linearized KdV

$$
u_{t}+c u_{x}+b u_{x x x}=0
$$

where $b$ and $c$ are constants.

$$
\begin{gathered}
w=k c-b k^{3} \quad w_{k}=c-3 b k^{2}, \quad w_{k k}=-6 b k, \quad w_{k k k}=-6 b \\
\Omega(\kappa)=-3 b k \kappa^{2}-b \kappa^{3}
\end{gathered}
$$

Thus, the envelope of the linearized KdV equation satisfies the equation

$$
A_{t}+i 3 b k A_{\zeta \zeta}+b A_{\zeta \zeta \zeta}=0
$$

The partial differential equation for the envelope of a weakly nonlinear wavepacket in the KdV equation will be identical except for additional terms, nonlinear in the envelope, which will be derived in the next section.

\section{Derivation of the NLS Equation from the KdV Equation}

The perturbation series approximates two independent processes: nonlinearity and dispersion. The most interesting regime is when these effects are of comparable importance. We shall therefore assume that nonlinearity and dispersion are of the same order of magnitude, thereby collapsing a double series into an expansion in a single parameter $\epsilon$. However, the resulting series will be accurate when nonlinearity $>>$ dispersion or vice versa so as long as the larger effect is sufficiently small.

The KdV equation is

$$
u_{t}+c u_{x}+a u u_{x}+b u_{x x x}=0
$$

where $a, b$, and $c$ are constants. To focus on weakly nonlinear wavepackets, assume that the lowest order solution is

$$
u(x, t) \sim \epsilon A(\zeta, \tau) \exp (i[k x-\omega(k) t])
$$

where $\epsilon<<1$ is the perturbation parameter, $k$ is the zonal wavenumber of the "carrier wave" whose frequency, phase and group velocities are specified by the linear $\mathrm{KdV}$ dispersion relation as

$$
\omega=k c-b k^{3} \quad \rightarrow \quad c_{p}(k)=c-b k^{2} \quad \& \quad c_{g}(k)=c-3 b k^{2}
$$

and where the "slow" space and time variables are

$$
\zeta \equiv \epsilon\left(x-c_{g}(k) t\right), \quad \tau=\epsilon^{2} t, \quad \tilde{\tau}=\epsilon^{3} t, \quad \bar{\tau}=\epsilon^{4} t
$$


The factor $A(\zeta, \tau)$ is the called the "envelope" and will evolve according to the NLS equation or its generalization. Different powers of $\epsilon$ (from the scalings of Eqs.15 and 17) also give legitimate limits, but a posteriori our choices turn out to be the ones such that nonlinearity and dispersion are of equal magnitude.

Through the method of multiple scales[7,5,1], one can show that the weakly nonlinear wavepacket has an asymptotic expansion of the form

$$
u=\epsilon \sum_{j=0}^{\infty} \epsilon^{j} \sum_{m=0}^{j+1} A^{j m}(\zeta, \tau) E^{m}+\text { c. с. }
$$

where $E$ is the "carrier wave":

$$
E(x, t) \equiv \exp (i[k x-\omega(k) t])
$$

where "c. c." denotes the complex conjugate of the terms in the sum.

For notational simplicity, write $A^{01}=A(x, t)$. The terms which are independent of the carrier wave are known collectively as the "long wave" components; the terms proportional to $E^{ \pm m}$ are the $m$-th harmonics except for $m=1$, which is the "fundamental" $A(x, t)$.

Derivatives are interpreted by applying the chain rule:

$$
\frac{\partial}{\partial t} \rightarrow \frac{\partial}{\partial t}-\epsilon c_{g} \frac{\partial}{\partial \zeta}+\epsilon^{2} \frac{\partial}{\partial \tau}+\epsilon^{3} \frac{\partial}{\partial \tilde{\tau}}+\epsilon^{4} \frac{\partial}{\partial \tilde{\tau}}, \quad \frac{\partial}{\partial x} \rightarrow \frac{\partial}{\partial x}+\epsilon \frac{\partial}{\partial \zeta}
$$

In a minor (and common) abuse of notation, the same symbols $x$ and $t$ are used for the "fast" space and time variables as for the original coordinates.

Matching powers of $\epsilon$ and the carrier wave exponential $E$ shows that at $O\left(\epsilon^{j+1} E^{m}\right), A^{j m}$ solves an equation of the form

$$
\left\{i k^{3} b m\left[1-m^{2}\right]\right\} A^{j m}(\zeta, \tau)=R^{j, m}
$$

where the expression in braces is $\left[\left(\partial_{t}+c \partial_{x}+b \partial_{x x x}\right) E^{m}\right] / E^{m}$ and where $R^{j+1, m}$ denotes the $O\left(\epsilon^{j} E^{m}\right)$ part of the "residual" function, which is the result of substituting the expansion into the $\mathrm{KdV}$ equation and then dividing by the common factor of $\epsilon$.

For $m=1$, i. e., the fundamental, the left-hand side of Eq. 21 is zero. Therefore, the perturbation scheme fails unless $R^{j, 1}=0$ for all $j \geq 0$. This set of "nonsecularity" conditions determines the linear dispersion relation at lowest order, regurgitates the usual linear definition of group velocity $\left(c_{g} \equiv\right.$ $\partial \omega(k) / \partial k)$ for $j=1$, and demands that $A$ evolve according to the NLS equa- 
tion to satisfy $R^{2,1}=0$. The next two orders give expressions for the time derivatives of $A$ on the very slow $(\tilde{\tau})$ and ultra-slow $(\bar{\tau})$ time scales.

The longwave components are independent of the "fast" variables; because derivatives with respect to the slow variable $\zeta$ are multiplied by $\epsilon, A^{j 0}$ appears in the residual only at one order higher in $\epsilon$ than the harmonics of the same magnitude in $\epsilon, A^{j m}, m>1$. The longwave components satisfy equations of the form

$$
3 b k^{2} A_{\zeta}^{j 0}=R^{j, 0}
$$

It turns out that after some manipulation, the longwave component of the residual can always be written as the derivative of an expression with respect to $\zeta$. This allows us to integrate with respect to $\zeta$ (trivially) and obtain an explicit expression for $A^{j 0}$.

The lowest non-trivial solution is to compute the $O\left(\epsilon^{2}\right)$ longwave and second harmonic components and then $R^{2,1}=0$ yields the NLS equation. This was previously derived in Appendix $\mathrm{C}$ of [1], closely following [5]. In this article, we extend the analysis two orders higher.

This introduces two minor technical complications. First, it is very helpful to remove time derivatives from the higher order residuals by replacing $A_{\tau}$ by invoking the NLS equation it solves. Second, for the longwave components, it is necessary to manipulate the simplified residual into the form of $\zeta$-derivative so that the expression for $A_{\zeta}^{j 0}$ can be integrated. This requires repeated use of identities such as $A A_{\zeta \zeta}^{*}=\left(A A_{\zeta}^{*}\right)_{\zeta}-A_{\zeta} A_{\zeta}^{*}$ where the asterisk denotes complex conjugation; formally integrating the residual in $\zeta$, these identities can be equivalently interpreted as repeated integration-by-parts.

Denoting the complex conjugate of $A$ by $A^{*}$, the corrections are

$$
\begin{gathered}
A^{10}=-\frac{a}{3 b k^{2}} A^{2}, \quad A^{12}=\frac{a}{6 b k^{2}} A^{2} \\
A^{20}=i \frac{a}{3 b k^{3}}\left\{A A_{\zeta}^{*}-A^{*} A_{\zeta}\right\}, \quad A^{22}=i \frac{a}{3 b k^{3}} A A_{\zeta}, \quad A^{23}=\frac{a^{2}}{48 b^{2} k^{4}} A^{3} \\
A^{30}=\frac{a}{3 b k^{4}}\left\{A A_{\zeta \zeta}^{*}+A^{*} A_{\zeta \zeta}-A_{\zeta} A_{\zeta}^{*}\right\}
\end{gathered}
$$




$$
\begin{gathered}
A^{32}=-\frac{a^{3}}{432 b^{3} k^{6}} A^{3} A^{*}-\frac{a}{6 b k^{4}} A_{\zeta}^{2}-\frac{a}{3 b k^{4}} A A_{\zeta \zeta} \\
A^{33}=i \frac{a^{2}}{12 b^{2} k^{5}} A^{2} A_{\zeta} \\
A^{34}=\frac{a^{3}}{432 b^{3} k^{6}} A^{4}
\end{gathered}
$$

At $O\left(\epsilon^{3}\right)$, one obtains the usual NLS equation:

$$
A_{\tau}+i 3 b k A_{\zeta \zeta}-i \frac{a^{2}}{6 b k} A|A|^{2}=0
$$

At higher order, one obtains variations with $A$ on longer timescales. The most convenient procedure is to simply combine these slow and ultra-slow time tendencies with the NLS equation to obtain

$$
\begin{gathered}
A_{\tau}+i 3 b k A_{\zeta \zeta}-i \frac{a^{2}}{6 b k} A|A|^{2} \quad[\text { Generalized-NLS }] \\
+b A_{\zeta \zeta \zeta}-\frac{a^{2}}{2 b k^{2}} A^{2} A_{\zeta}^{*}-\frac{a^{2}}{3 b k^{2}}|A|^{2} A_{\zeta} \\
+i \frac{a^{4}}{864 b^{3} k^{5}}|A|^{4} A+i \frac{2 a^{2}}{3 b k^{3}} A^{2} A_{\zeta \zeta}^{*} \\
\quad-i \frac{a^{2}}{6 b k^{3}}\left(A_{\zeta}\right)^{2} A^{*}+i \frac{a^{2}}{3 b k^{3}} A_{\zeta} A_{\zeta}^{*} A=0
\end{gathered}
$$

where the second line is the correction at $O\left(\epsilon^{4}\right)$ and the last two lines are that at $O\left(\epsilon^{5}\right)$, respectively. (Our approximation, (23-29) above removes the longwave and fundamental parts of the residual at $O\left(\epsilon^{5}\right)$, but not the higher harmonics.)

The perturbative solutions at various orders will be compared with exact $\mathrm{KdV}$ solutions in the next-to-last section below.

\section{Defocusing}

The NLS equation has two qualitatively different cases which cannot be converted into one another by any rescaling of space, time or amplitude. When the signs of the dispersive $\left(A_{\zeta \zeta}\right)$ and nonlinear terms are the same, nonlinearity and dispersion can balance so as to create an envelope solitary wave. 
When derived from the KdV equation, the signs of the dispersive coefficient, $-3 b k$, and the nonlinear coefficient, $a^{2} /(6 b k)$, are always opposite independent of the signs and magnitude of the coefficients $a$ and $b$ of the KdV equation. Envelope solitary waves are impossible (although the KdV equation certainly has solitary waves consisting of a single crest) and dispersion and nonlinearity both act to widen the envelope, at least in the asymptotic limit as $t \rightarrow \infty$. (The envelope may compress for a finite time interval if the initial conditions are appropriately contrived, but will always widen and shrink in amplitude for sufficiently large time.)

\section{$5 \quad$ Perturbation Parameters}

There is some arbitrariness in the definitions of the perturbation parameters because phrases like "width of the envelope" are meaningful only in order-ofmagnitude. Nevertheless, the following choices are reasonable. The dispersion parameter $\lambda$ is the ratio of the length scale of the carrier wave, $1 / k$, to the width $1 / \beta(t)$ of the envelope:

$$
\lambda(t) \equiv \beta(t) / k \quad \text { [Dispersion Parameter] }
$$

The nonlinearity parameter will be defined to be the ratio of the largest component of the first order solution to the maximum of the fundamental.

$$
\mu(t) \equiv \max _{x}|A(x, t)| /\left(3 b k^{2}\right)
$$

For sufficiently large time, as discussed below, the wavepackets of the KdV equation are always widening, implying that $\beta(t) \sim O(1 / t), t \rightarrow \infty$ is a decreasing function of time. To conserve the total energy of the wavepacket, the envelope must simultaneously decrease as $O(1 / \sqrt{t})$. [This seems a bit counterintuitive, but recall that the total, integrated energy, which must be constant in time, is proportional to the square of the amplitude but is linear in the width of the envelope.] The ratio of the nonlinear to dispersive terms in the NLS equation is

$$
\frac{\text { nonlinear }}{\text { dispersive }} \sim\left(\frac{\mu^{2}}{\lambda^{2}}\right) \sim O(t), \quad t \rightarrow
$$

Thus, the envelope evolves more and more slowly as time increases, but such evolution as there is becomes increasingly dominated by nonlinearity. 


\section{$6 \quad \mathrm{KdV}$ from NLS}

The NLS equation is usually derived by perturbation theory from a system of equations far more complicated than the KdV equation. However, if that more complicated system can be approximated in the longwave limit by the KdV equation, then the $\mathrm{KdV}$ equation must be consistent with the limit $k \rightarrow 0$ of the NLS equation. It follows that if we denote the nonlinear coefficient of the NLS equation by $\nu(k)$, then

$$
\nu(k) \sim \frac{a^{2}}{6 b k} \quad \leftrightarrow a=\sqrt{\lim _{k \rightarrow 0}\{6 b k \nu(k)\}}
$$

where $a$ is the coefficient of the nonlinear term of the KdV equation. Thus, the "Landau constant" $\nu(k)$ always has a simple pole at $k=0$ when the longwave $(k \rightarrow 0)$ limit is described also by the KdV equation.

The dispersive coefficient of the KdV equation must be consistent with the linear dispersion relation for both the underlying system and the KdV equation itself and is therefore always

$$
b=-(1 / 6) \omega_{k k k}(k=0)
$$

where $\omega_{k k k}$ denotes the third derivative of the frequency with respect to wavenumber. The expression for the nonlinear coefficient of the KdV equation is thus completely determined by the longwave limits of the linear dispersion relation and of $\nu(k)$ and can be rewritten

$$
a=\sqrt{-\omega_{k k k}(0) \lim _{k \rightarrow 0}\{k \nu(k)\}}
$$

Thus, if one has a computer program to evaluate $\nu(k)$, then it is not necessary to write separate software or redo the usual KdV derivation to obtain the coefficients of the KdV model. The dispersive and nonlinear coefficients of the $\mathrm{KdV}$ equation are always given by (35) and (36).

Marshall and Boyd[8], who illustrate this principle for equatorially-trapped wave in the ocean, show something even stronger is true. Wave packets exhibit other kinds of resonances including longwave/short wave resonances and second harmonic resonances. These, too, generate poles in the Landau constant as a function of $k$, and the coefficients of the "long wave/short wave" system and of the "second harmonic" system fall out from the linear dispersive theory plus the residue of $\nu(k)$ at the resonance pole.

The mathematics of the pole of the Landau constant at $k=0$ is clear, but what is the physics? The answer is that as $k \rightarrow 0$, the phase speeds and 
group velocities of the second harmonic (wavenumber $=2 k$ ) and the longwave (wavenumber zero) differ from that of the fundamental (wavenumber $=k$ ) only by $O\left(k^{2}\right)$. Thus, as $k \rightarrow 0$, these three components are increasingly close to resonance. This resonance in turn forces the Landau constant to grow as $k \rightarrow 0$.

There is one mildly misleading aspect to the pole: the amplitudes of the higher order terms in the multiple scales series increase inversely with $k$, too. Thus, the timescale for nonlinear interaction does not decrease to zero, but rather the perturbation expansion collapses, with higher terms as large as the fundamental, for sufficiently small $k$.

\section{$7 \quad$ Weakly Dispersive Waves}

The failure of the NLS perturbation theory as $k \rightarrow 0$ is rather disconcerting because the $\mathrm{KdV}$ equation is usually derived as a longwave approximation. Thus, there is only a narrow range of small $k$ where the wavenumber of the carrier wave of the wavepacket is sufficiently small that the KdV equation is a good model, and simultaneously so large that the NLS model is accurate. The NLS/KdV connection would seem to be much ado about a very narrow parameter range.

However, the equatorial Kelvin wave in the ocean, which was the target of our investigation [2], is quite different. In the absence of a mean flow, the Kelvin wave is nondispersive. Latitudinal variations in the mean east-west ocean currents induces a weak dispersion in the Kelvin mode, but to second order in the strength of the shear, the dispersion relation is quadratic in the east-west wavenumber. It follows that the third derivative term in the $\mathrm{KdV}$ equation captures the correct dispersive behavior for the Kelvin wave even when the wavenumber is not small.

One consequence is that $\mathrm{KdV}$-type Kelvin solitary waves are not restricted to long zonal scales (highly anisotropic solitons, much wider so that the solitons are highly anisotropic, much wider east-west than north-south); the KdV equation is a good approximation in describing "round" solitons of equal longitudinal and latitudinal width. A second consequence is that the description of the wavepackets of the oceanic equatorial Kelvin wave is captured for the

entire interesting range of parameters by deriving the NLS equation from the corresponding KdV approximation.

Similar comments apply to other species of waves. When the waves are nearly nondispersive, the KdV approximation will apply to moderate as well as long length scales. The NLS approximation derived from the KdV equation will 
similarly have a wide range of applicability.

\section{Numerical Experiments}

Although we experimented with many variations in amplitude and width of the envelope, we shall discuss one representative case which evolved from the initial condition

$$
u(x, 0)=6 \mu \operatorname{sech}(\lambda x) \cos (x+\pi / 3)
$$

where the nonlinearity and dispersion parameters are $\mu=1 / 10$ and $\lambda=1 / 10$. The domain has periodic boundary conditions where the period $P=128 \pi$. The Generalized-NLS with quintic nonlinearity and also the KdV equation were solved by a Fourier pseudospectral method with 1024 grid points, combined with a fourth order Runge-Kutta time marching method with a time step of $1 / 400$ on the interval $t \in[0,200]$. The high spatial and temporal resolution for the Generalized-NLS equation is, except for ease of programming, quite unnecessary; the whole point is that the envelope $A(\zeta, \tau)$ varies slowly with both space and time.

Fig. 1 compares the initial and final wavepackets, showing that the amplitude has decreased to almost exactly one-third of its initial value. The width has increased by roughly the square of the reciprocal of the amplitude decrease, approximately a factor of nine. The packet has become so wide that, thanks to the periodic boundary conditions, packets centered on neighboring periods are now overlapping. The scalloping or wiggliness of the envelopes near the edges of the periodicity interval at $t=200$ is the spoor of this overlap-ofpackets; we have done many experiments with both the GNLS and standard NLS equations, and this wiggliness-at-the-edges always seems to develop when the packet widens beyond the edges of the periodicity interval.

Fig. 2 shows the evolution in the errors of the Fourier coefficients with time. The solution of the quintic Generalized NLS equation was (inconsistently) used to evaluate the perturbative solutions at each order. Zeroth order included only $A(\zeta, \tau)$, first order added $A^{10}$ and $A^{12}$, and so on. Clearly, the method of multiple scales has been successful with good uniformity in time.

Fig. 3 compares the initial and final Fourier coefficients with the predicted Fourier coefficients. The predicted fourth harmonic (rightmost dashed bump) is an order of magnitude too small, but all the higher harmonics have decreased considerably over time both in the perturbative prediction and in the true KdV solution. The very dramatic narrowing of the longwave component, which is the leftmost bump, is also predicted with quantitative accuracy by perturbation theory. 
Fig. 4 compares the absolute values of the final coefficients of the KdV solution with the errors in absolute value of the third order perturbative prediction. Another intriguing surprise is that the absolute errors in Fourier coefficients are largest for the fundamental (that is, in the wavenumber range $k / 2$ to $(3 / 2) k$ where here $k=1$ ) where the relative errors (about 1 part in 10,000) are smallest. For the fourth harmonic and above, the relative error is very large. Overall, though, the perturbation theory does well for this case where the perturbation parameters are 1/10.

Although the dramatic narrowing (in wavenumber space) of the longwave component is well-captured by the perturbation theory, we have not been able to arrive at a simple heuristic explanation. Perhaps the reader will be more ingenious!

Similarly, we do not understand why the fourth harmonic is an order of magnitude larger than predicted by perturbation theory, nor why it dips to near-zero amplitude at a wavenumber of $4 k$, right in the center of the harmonic.

We performed the usual experiments, halving the timestep and also independently doubling the width of the spatial domain, but these puzzling features were unaltered.

\section{Summary}

We have shown the following:

- The NLS equation derived from the KdV equation is always the defocusing case; envelope solitary waves cannot form in the KdV solution.

- NLS perturbation theory is here explicitly extended to two orders beyond the NLS equation itself.

- The Generalized-NLS equation, which describes the evolution of the envelope, is quintically nonlinear and third order in space.

- A special quirk of the KdV equation is that the Generalized-NLS, when carried to one order beyond the usual NLS, captures the linear dispersion of the KdV equation exactly. For packets of finite amplitude, however, there is some small error because the nonlinear terms cannot exactly represent the nonlinearity of the KdV equation.

- The nonlinear coefficient of the NLS equation has a pole in $k$ as the wavenumber $k$ of the carrier wave tends to zero.

- The residue of this pole gives an explicit expression for the nonlinear coefficient of the KdV equation and vice-versa.

- When the underlying waves are only weakly dispersive, as true of equatorial Kelvin waves in the ocean, the $\mathrm{KdV}$ equation is an accurate model even for 
waves of moderate or short length scale. The NLS-from-KdV theory then has a similarly large region of validity.

- Our numerical experiments show good agreement between the NLS perturbation theory and the exact $\mathrm{KdV}$ wavepacket for small and moderate times.

Nevertheless, a number of open questions remain including:

(1) Does the Generalized-NLS equation have solitary waves in some parameter range? [It certainly is soliton-free when the higher order terms can be neglected compared to those that appear also in the NLS equation.]

(2) How does the Generalized-NLS equation, derived from the KdV equation, compare to similar but lower order equations derived from more complex systems by Dysthe[3] (water waves), Stiassnie[9] (Zakharov equation) and Hogan[4] (capillary-gravity water waves) ?

(3) Is there a simple hand-waving or heuristic explanation that makes it obvious, instead of merely true, that the $\mathrm{KdV}$ dynamics always gives nonlinear defocusing of wave packets?

\section{Thick: $2|A(x, 0)|$ Thick Dashed: $2|A(x, 200)|$ Thin: KdV solution}

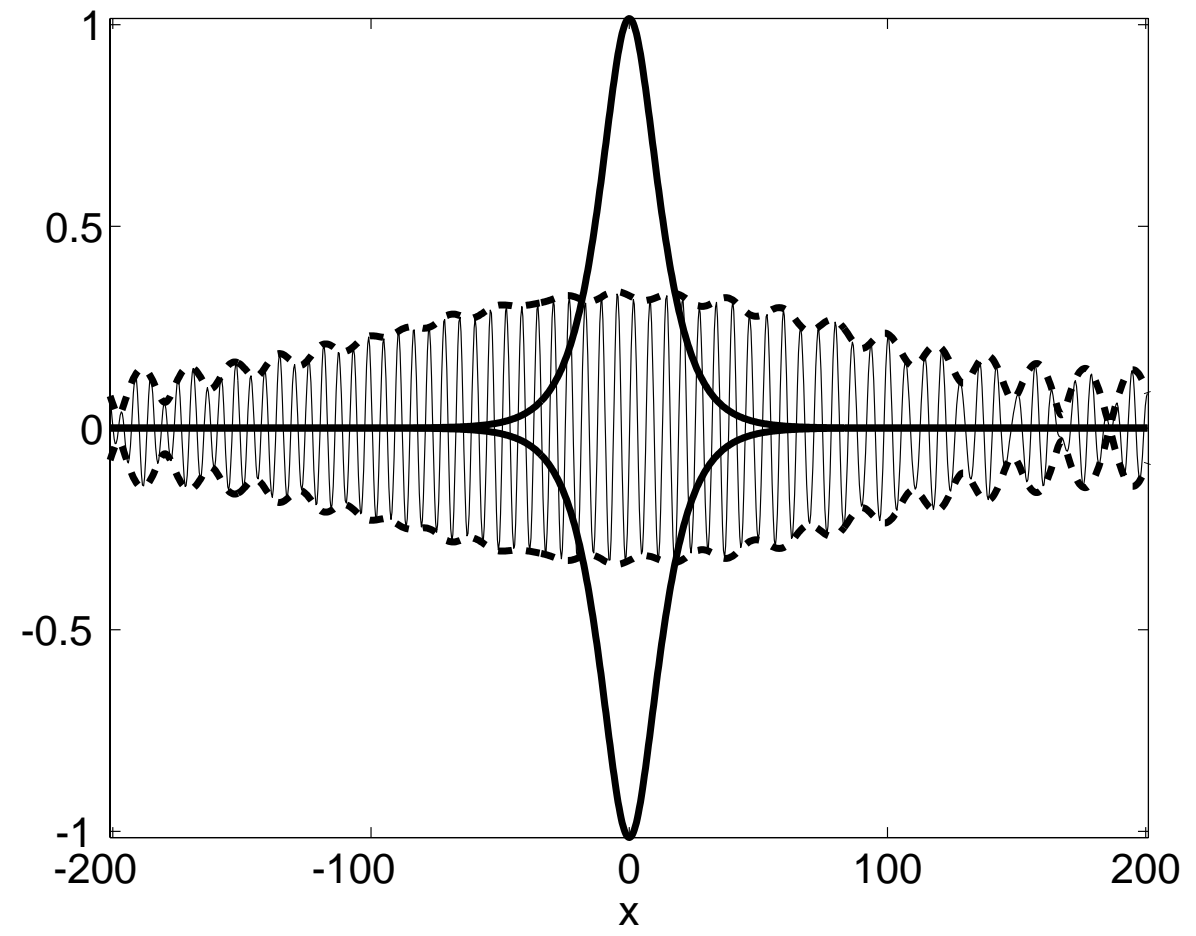

Fig. 1. The tall solid curve is the initial envelope. (Because the initial solution is $A \exp (i k x)+A^{*} \exp (-i k x)$, we show twice $\left.|A(x, 0)|.\right)$ The thick dashed curve is the envelope at $t=200$. The thin curve is the $\mathrm{KdV}$ solution at $t=200$. The predicted solution was also plotted, but is graphically indistinguishable from the exact solution. All plots were scaled by dividing by the maximum initial value of the KdV solution. 
(4) Why is the fourth harmonic approximated rather badly for large times when lower (and larger amplitude) Fourier components are approximated well?

(5) Why does the longwave component, when its Fourier coefficients are plotted, narrow so drastically with increasing time while the fundamental and higher harmonics become a little wider?

We thank the reviewer for useful comments.

\section{References}

[1] J. P. Boyd, Equatorial solitary waves, Part II: Envelope solitons, J. Phys. Oceangr., 13 (1983), pp. 428-449.

[2] G.-Y. Chen And J. P. Boyd, Nonlinear wave packets of equatorial Kelvin waves, Dyn. Atmos. Oceans, (2000). In preparation.

[3] K. B. Dysthe, Note on a modification to the nonlinear Schroedinger equations and their solutions, Proc. Roy. Soc. London A, 369 (1979), pp. 105-114.

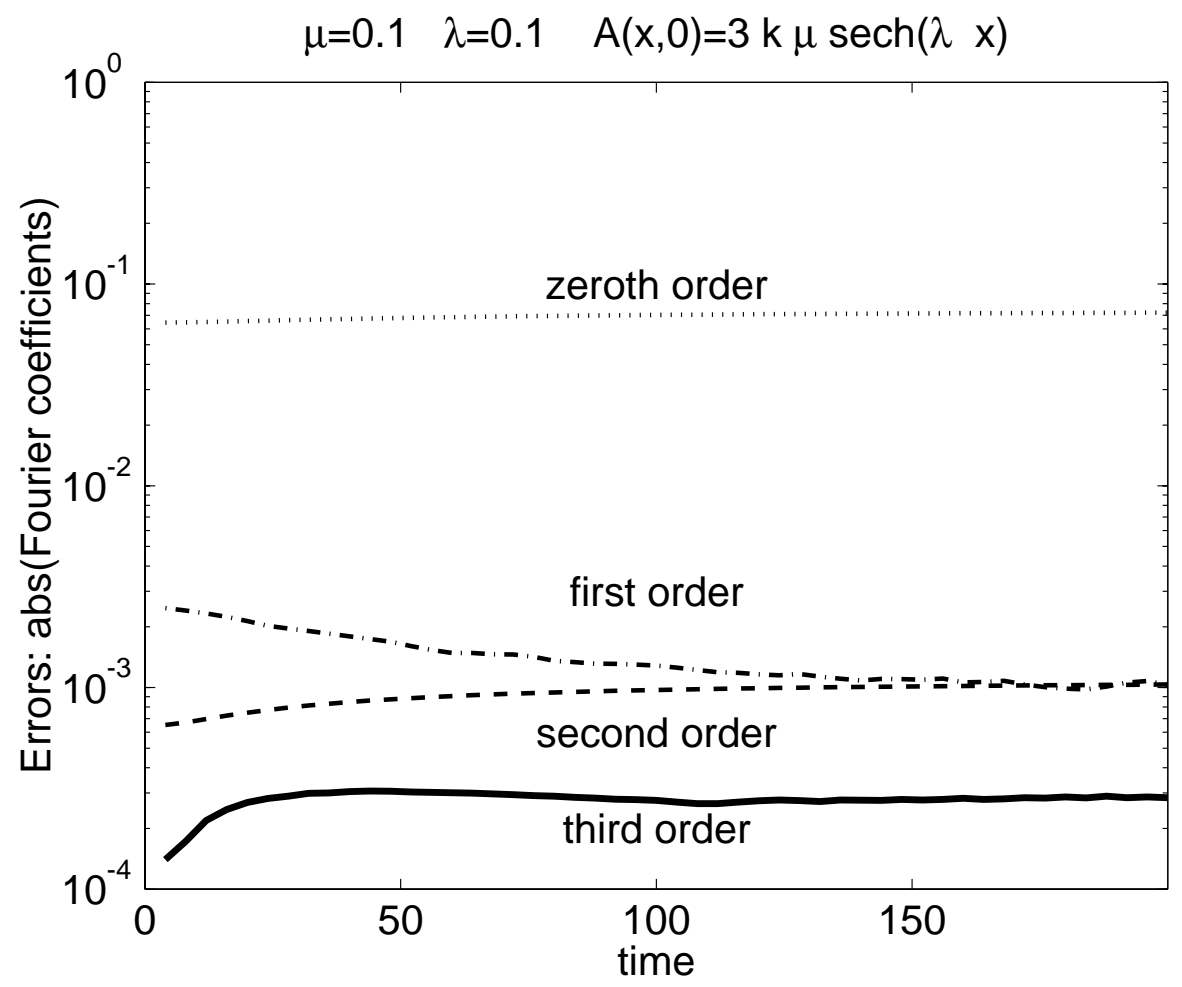

Fig. 2. The curves are the maximum differences in the absolute values of the Fourier coefficients of $u_{K d V}$ and $u^{(j)}$. The errors have been scaled by dividing by the maximum Fourier coefficient at each time. 
[4] S. J. Hogan, The fourth-order evolution equation for deep-water gravitycapillary waves, Proc. R. Soc. London Ser. A, 402 (1985), pp. 359-372.

[5] R. S. Johnson, Nonlinear, strongly dispersive water waves in arbitrary shear, Proceedings of the Royal Society of London A, 338 (1976), pp. 101-114.

[6] R. S. Johnson, A Modern Introduction to the Mathematical Theory of Water Waves, vol. 18 of Cambridge Texts in Applied Mathematics, Cambridge University Press, Cambridge, 1997.

[7] J. Kevorkian and J. D. Cole, Multiple Scale and Singular Perturbation Methods, Springer-Verlag, New York, 1996.

[8] H. G. Marshall And J. P. Boyd, Solitons in a continuously stratified equatorial ocean, Journal of Physical Oceanography, 17 (1987), pp. 1016-1031. Hermite function application.

[9] M. Stiassnie, Note on the modified nonlinear Schroedinger equation for deep water waves, Wave Motion, 6 (1984), pp. 431-433.

[10] E. R. Tracy, J. W. Larson, A. R. Osborne, and L. Bergamasco, On the Nonlinear Schroedinger limit of the Korteweg-deVries equation, Physica D, 32 (1988), pp. 83- 106.

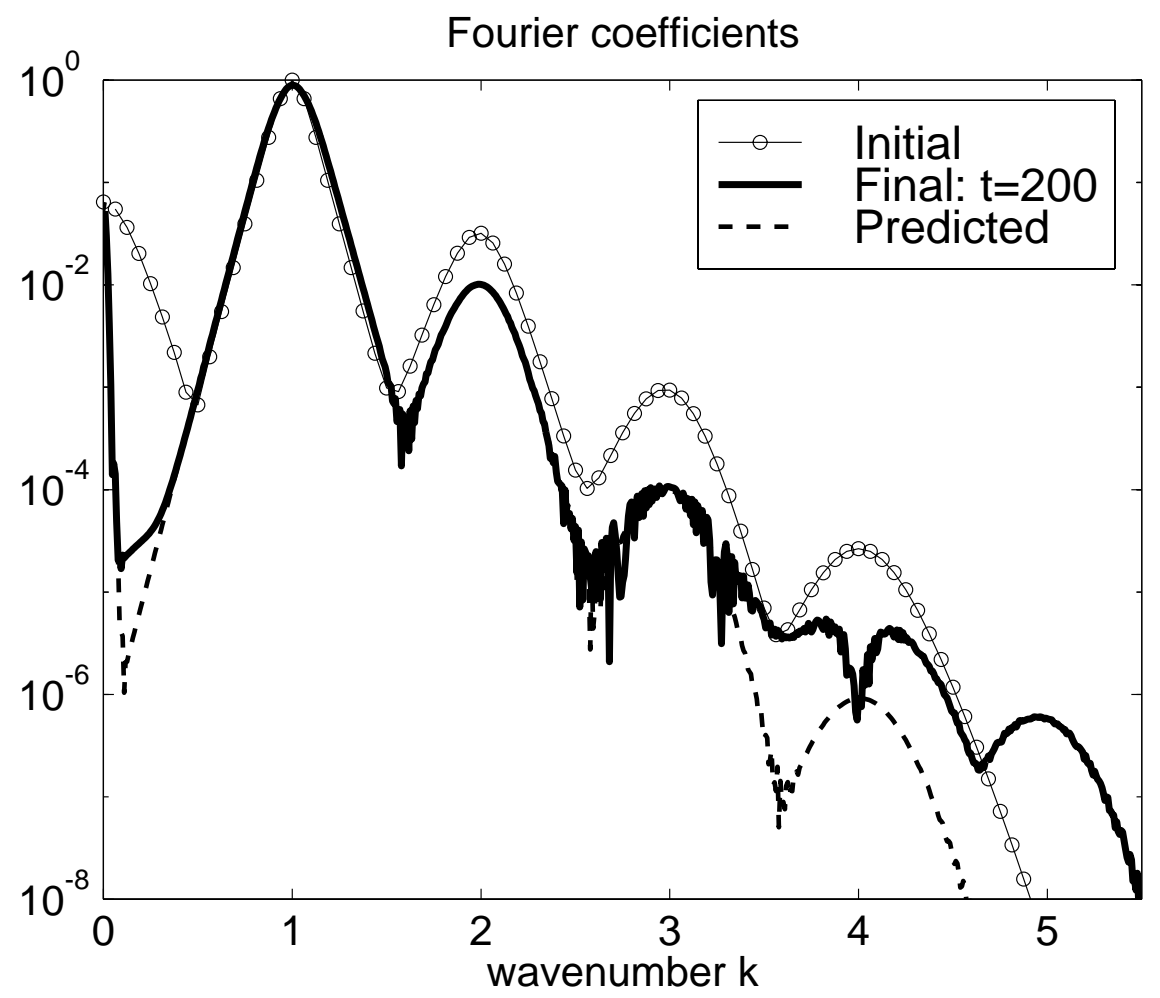

Fig. 3. The absolute values of the initial and final Fourier coefficients are the thin line with circles and the thick solid line, respectively. The predicted coefficients are also shown as the dashed curve, but are difficult to distinguish from the exact coefficients except under the fourth and fifth harmonics (wavenumber $>3.5$ ). 
[11] _ The relationship between the spectral theories for the periodic KortewegdeVries and Nonlinear Schroedinger equations, in Nonlinear Topics of Ocean Physics: Fermi Summer School, Course LIX, A. R. Osborne, ed., North-Holland, Amsterdam, 1991, pp. 769-825.

[12] G. B. Whitham, Linear and Nonlinear Waves, John Wiley \& Sons, New York, 1974.

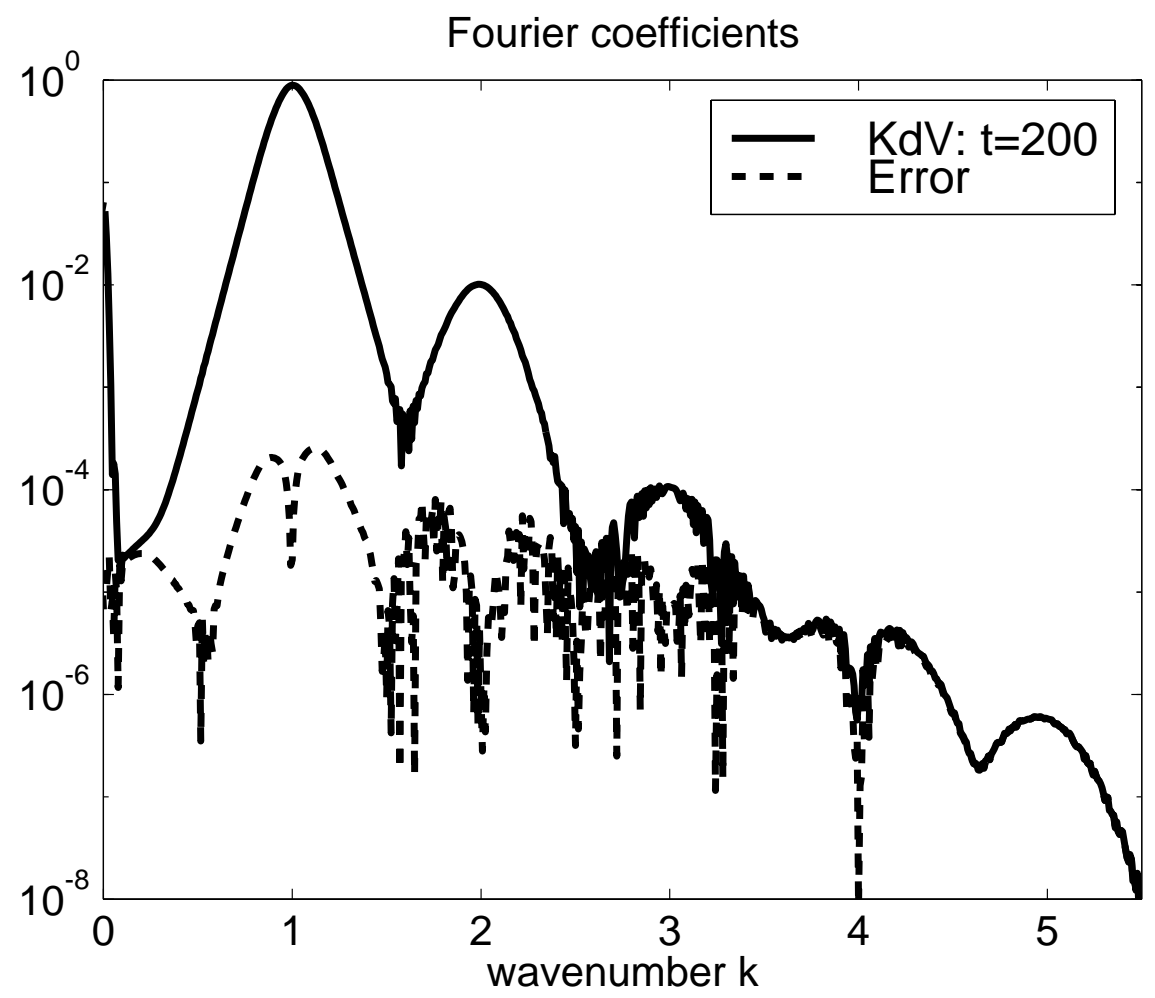

Fig. 4. The thick solid line is the absolute value of the Fourier coefficients of the KdV solution at the final time, just as in the previous figure. The lower curve (dashed) is the difference between the absolute values of the coefficients of the KdV solution and the prediction of third order perturbation theory. Note that for the fourth harmonic $(k \in[-3.5,4.5])$ and larger wavenumber, the error merges with the coefficient curve so that the relative error for the fourth and higher harmonics is close to $100 \%$. 